\title{
Synthesis of Oxazolidinone Phosphonate Derivatives, Part I
}

\author{
Jae-Min Hwang, Woon-Seob Shin,; and Kang-Yeoun Jung ${ }^{*}$ \\ Department of Environmental \& Applied Chemistry, College of Engineering, \\ Kanghtng National Lniversity, Kanghung 210-702, Korea \\ -Department of Horobiolog, Kwandong Lniversity College of Hedicine, Kangning 210-701, Korea \\ Received.April 28, 2004
}

\begin{abstract}
Several new derivatives of oxazolidinone phosphonate. that are expected to show biological activity, were prepared efficiently by previously published methods. $\gamma$-Ketophosphonate 1 was synthesized using pentacovalent oxaphosphorane chemistry followed by reductive amination with aromatic amine of oxazolidinone 4. Biological activity of the synthetic compounds has been studied. One of the șynthetic compounds showed promising result for us to pursue further studies.
\end{abstract}

Key Words: Oxazolidinone. Oxazolidinone phosphonates. Antibacterial agent. N-Arỵlation $\nsucc$ Ketophosphonate

\section{Introduction}

Oxazolidinones are very important class of antibacterial agents. ' which are active against Gran-positive human pathogens including drug resistant strains MRSA (methicillin-resistant Staphlococcus awreus). ${ }^{2}$ MRSE (methicillinresistant Staphtococcus epidermis). ${ }^{3}$ and VRE (vancontycinresistant Enterococi $i)^{+}$as well as selected anaerobic organismis through a unique mechanism of action. To date many groups have reported the synthesis and biological activity of novel oxazolidinones since a few of synthetic oxazolidinone derivatives had shown antibacterial activity The first oxazolidinone, linezolid (Zyox ${ }^{\mathrm{TM}}$ ) is now being marketed for the treatment of nulti-drug resistant Grampositive infections. sc $^{\text {s }}$

Benzoxazolone moiety in many drugs has demonstrated to be very important group for the treatments of various disease $^{6}$ and likewise. aninophosphonic acids which exist in many natural products, play very important role in biological system. ${ }^{7}$ Our idea was to combine aninophosphonate moiety with variously substituted benzoxazolones giving access to a wide array of structures. which can be expected to show interesting biological and pharmacological properties

We. therefore. selected the aminophosphonate group as a potential replacement for general substituents such as alcohol or carboxylic acid in our designed compounds. and report the preliminary sỵthesis of oxazolidinone phosphonate derivatives.

\section{Results and Discussion}

Synthetic route to target compounds is summarized in Scheme 1. $\gamma$-Ketophosphonate 1 was obtained using previously developed method ${ }^{8}$ in excellent yield. Aromatic amine 4 derived from commercially available 2-amino-5-nitrophenol in three steps (amine protection with $\mathrm{CBZCl}$ cyclization. and reduction) was coupled with 1 to give the reductive amination product $\mathbf{5}$. Reductive anination method used in this coupling reaction was previously reported. ${ }^{9}$

Regioselective arylation on the nitrogen of the benzoxazolone compound 5 was performed to give the desired derivatives, 6a-c, in reasonable yields. This regioselective arylation was confimed by NMR data comparison of compound 5 with compounds 6 a-c.

Anino products, $7 \mathrm{a}-\mathrm{c}$, were obtained by the reduction of nitro compounds. 6a-c. with $\mathrm{H}_{2}$ and anmoniumformate. $\gamma$ Aminophosphonates, 7a-c, were treated with TMSBr to give $\gamma$-aminophosphonic acids, 8a-c for biological studies.

Biological activity of synthetic compounds was determined by 2-fold agar dilution method followed by procedure of National Committee for Clinical Laboratory Standards (NCCLS). ${ }^{\text {li }}$ Standard strains of $E$. coll ATCC 25922. S. aureus ATCC 25923 and $P$. antoginosa ATCC 27853 were used for the determination of antibacterial activity. Each compound was dissolved in methanol and added into sterilized Mueller-Hinton agar in 2-fold concentrations ( 0 to $500 \mu \mathrm{g} / \mathrm{mL}$ ) prior to pouring into plates. After solidification. fresh bacterial suspensions were prepared in sterile saline and adjusted to an optical density of $0.2-0.3$ at $600 \mathrm{~mm}$. Adjusted inoculum $(10 \mu \mathrm{L})$ was spotted on each plate Plates were incubated for 24 to $48 \mathrm{~h}$ at $37^{\circ} \mathrm{C}$ in ambient air. Preliminary biological data are shown below in Table 1

In conclusion, several new compounds were synthesized efficiently by use of pentacovalent $\mathrm{P}(\mathrm{V})$ oxaphosphorane chemistry and reductive amination. All of the analogues were tested in vitro against a panel of Gram-positive and Gram-negative bacteria. Although the biological activity was not very promising compared to oxaciline. it allows us investigate other derivatives to obtain a better result in the future. Modification of the compound $\mathbf{8 b}$. particularly at the two amino groups and $\alpha$ position to the phosphonate group. will be studied. 

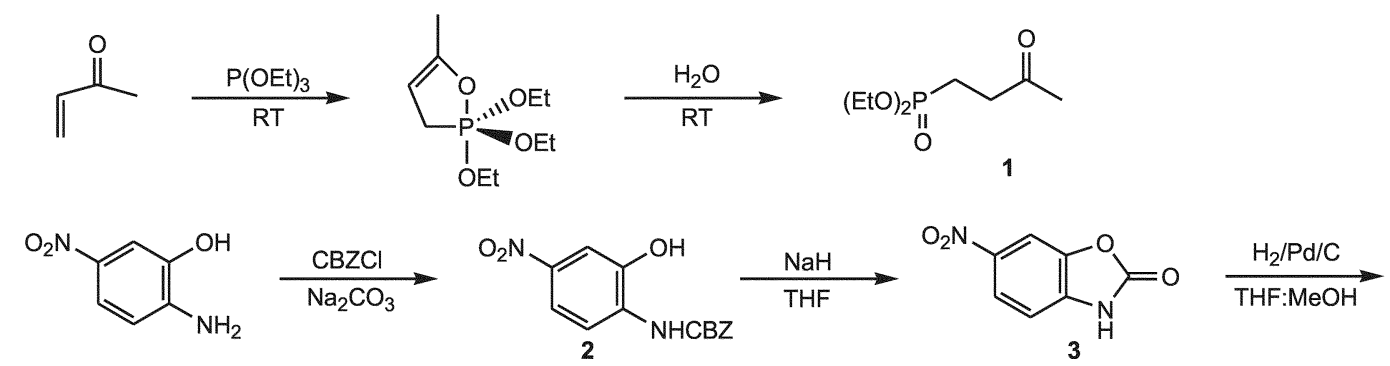<smiles>Nc1ccc2[nH]c(=O)oc2c1</smiles><smiles>CCOC(=O)CP(=O)(CCC(C)Nc1ccc2[nH]c(=O)oc2c1)OCC</smiles>

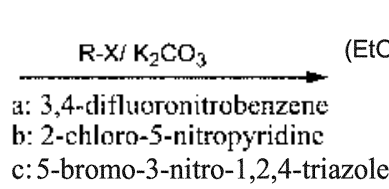<smiles>[R]n1c(=O)oc2cc(NC(C)CCP(=O)(O)OCC)ccc21</smiles>

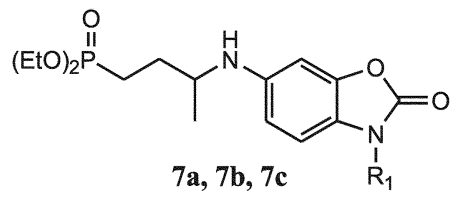<smiles>CCCC</smiles>

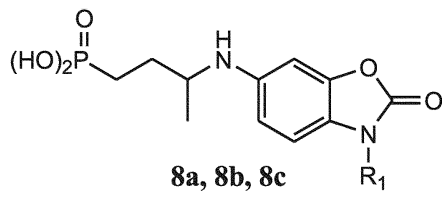

\begin{tabular}{|c|c|c|c|}
\hline Compounds & $\mathbf{a}$ & b & c \\
\hline 6 & & & \\
\hline$R$ & & & \\
\hline
\end{tabular}

Scheme 1. Synthesis of Oxarolidinone $\gamma$-Aminophosphonic Acids.

Table 1. Minimum Inhibitory Concentration of Sy nthelic Compounds

\begin{tabular}{lccccc}
\hline \multirow{2}{*}{ Organisms } & \multicolumn{5}{c}{ MIC $(\mathrm{mg} / \mathrm{mL})^{*}$} \\
\cline { 2 - 6 } & $\mathbf{8 a}$ & $\mathbf{8 b}$ & $\mathbf{8 c}$ & $\mathbf{7 a}$ & Oxacilin \\
\hline S. andeus ATCC 25923 & $>500$ & $>125$ & $>500$ & $>500$ & 0.78 \\
E. coli ATCC 25922 & $>500$ & $>500$ & $>500$ & $>500$ & $>500$ \\
D. aurogmosa ATCC 27853 & $>500$ & $>500$ & $>500$ & $>500$ & $>500$ \\
\hline
\end{tabular}

*The minimum inhibitory concentration (MIC) wats defined as the lowest concentration of symthetic compounds in agar plates showing no visible bacterial growth.

\section{Experimental Section}

General. Dichloromethane and $E t_{i} N$ were distilled from $\mathrm{CaH}_{2}$ immediately prior to use. All non-aqueous reactions were conducted in flame-dried glassware, under an atmosphere of argon, with magnetic stirring. NMR spectra were obtained on a JOEL Lainda 300 spectrometer and recorded at 300 $\mathrm{MHz}$ for ${ }^{1} \mathrm{H}\left(75 \mathrm{MHz}\right.$ for $\left.{ }^{1}{ }^{1} \mathrm{C}\right)$ with $\mathrm{CDCl}_{3}$ as solvent and $\left(\mathrm{CH}_{3}\right)_{4} \mathrm{Si}\left({ }^{\mathrm{l}} \mathrm{H}\right)$ or $\mathrm{CDCl}_{3}\left({ }^{1} \mathrm{C}, 77.0 \mathrm{ppm}\right)$ as internal standards unless otherwise noted. All ${ }^{31} \mathrm{P}$ NMR chemical shifts are reported in ppm relative to $85 \% \mathrm{H}_{3} \mathrm{PO}_{4}$ (external standard). FI-IR spectra were recorded on a JASCO FR-IR 460 series. High resolution $\mathrm{FAB}$ mass spectra were obtained from the Hybrid LC-Quarapole-1OF Tandem Mass Spectrometer at the Kangnung National University.

6-Amino-3 $\boldsymbol{H}$-benzoxazol-2-one 4: A flame-dried $250 \mathrm{ml}$. round-bottom flask under argon was charged with benzoxazolone 3 (4.3 g. $23.93 \mathrm{mmol}$ ) and anhydrous mixture of THF : $\mathrm{MeOH}(35: 65,100 \mathrm{~mL})$. To this solution was added amınonium formate $(0.23 \mathrm{~g}, 3.7 \mathrm{mmol}$ ). After bubbling $\mathrm{Ar}$ through the reaction solution for $30 \mathrm{~min}$. Pd/C (catalyst 1.39 $\mathrm{mg} .0 .014 \mathrm{mmol}$ ) was added quickly. The reaction mixture were filtered after $3 \mathrm{hr}$ and concentrated in vacuo to give the crude product. After removal of the solvent under reduced pressure, the crude oil was purified by flash column chromatography using a gradient solvent system with methylene chloride and methanol to give the desired product $4(3.5 \mathrm{~g}, 23.32 \mathrm{mmol}, 97 \%)$. ${ }^{1} \mathrm{H}$ NMR (DMSO) $\delta 11.06$ (s. lH), $6.72(\mathrm{~d}, J=7.8 \mathrm{~Hz}, \mathrm{lH}), 6.48(\mathrm{~d}, J=1.8 \mathrm{~Hz}, 1 \mathrm{H}), 6.33$ (dd, $J=7.8,1.8 \mathrm{~Hz}, 1 \mathrm{H}), 4.98(\mathrm{~s}, 2 \mathrm{H}){ }^{1}{ }^{1} \mathrm{C}$ NMR $\delta 154.74$. $144.60,144.37,119.86,109.92,109.01,96.13$; HRFABMS calcd for $\mathrm{C}_{7} \mathrm{H}_{6}, \mathrm{~N}_{2} \mathrm{O}_{2}(\mathrm{M}-1)^{-}: 151.0429$, found: 151.0424 .

[3-(2-Oxo-2,3-dihydro-benzoxazol-6-yl)aminobutyl]phosphonic acid diethyl ester 5: A flame-dried $100 \mathrm{~mL}$ round-bottom flask under argon was charged with phosphonate $1(0.53 \mathrm{~g} .2 .55 \mathrm{mmol})$ and anhydrous THF $(20 \mathrm{~mL})$. To this solution was added sodium tri-acetoxy borohydride (0.96 g, $4.53 \mathrm{mmol})$ and $\mathrm{AcOH}$ (153 $\mathrm{mg}, 2.55 \mathrm{mmol})$. After being stirred for $5 \mathrm{~min}$ at room temperature, benzoxazolone 
$4(0.38 \mathrm{~mL} .2 .55 \mathrm{mumol})$ was added quickly. Reaction mixture was allowed to stir for $27 \mathrm{hr}$ and quenched with $1 \mathrm{~N}$ $\mathrm{NaOH}$. This aqueous mixture was extracted with $\mathrm{CH}_{2} \mathrm{Cl}_{2}$ and then the combined organic extracts were dried over anhydrous $\mathrm{Na}_{2} \mathrm{SO}_{4}$. After removal of the solvent under reduced pressure, the crude oil was purified by flash colunn chromatography using a gradient solvent system with methylene chloride and methanol to give the desired product 5 (0.5 g. 1.45 nmiol. 57\%). ${ }^{\text {H NMR } 9.74 ~(s, ~ I H), ~} 6.79$ (d, $J$ $=8.1 \mathrm{~Hz} \cdot \mathrm{lH}) .6 .50(\mathrm{~d}, J=2.4 \mathrm{~Hz}, \mathrm{lH}) .6 .36(\mathrm{dd} . J=8.4 .2 .1$ $\mathrm{Hz}, 1 \mathrm{H}) .4 .11(\mathrm{~m}, 4 \mathrm{H}), 3.49(\mathrm{~m} .1 \mathrm{H}), 1.86$ (n. $4 \mathrm{H}) .1 .28$ (dt. $J=3.9,7.2 \mathrm{~Hz} .6 \mathrm{H}) .1 .20(\mathrm{~d}, J=6.2 \mathrm{~Hz}, 3 \mathrm{H}):{ }^{13} \mathrm{C}$ NMR $\delta$ 156.00. 145.21. 143.68. 120.68. 110.36. 109.08, 96.01. 61.78 (d. $\left.J_{C . \mathrm{P}}=6.2 \mathrm{~Hz}\right) .49 .42$ (d. $J_{C . \mathrm{P}}=16.1 \mathrm{~Hz}$ ). 29.11 (d. $\left.J_{\mathrm{C} \cdot \mathrm{P}}=4.3 \mathrm{~Hz}\right) .21 .98\left(\mathrm{~d} . J_{\mathrm{C} . \mathrm{P}}=140.6 \mathrm{~Hz}\right), 20.38 .16 .40\left(\mathrm{~d} . J_{\mathrm{C} . \mathrm{P}}\right.$ $=6.2 \mathrm{~Hz}) ;{ }^{31} \mathrm{P}$ NMR $\delta 32.7$; HRFABMS calcd for $\mathrm{C}_{15} \mathrm{H}_{23} \mathrm{~N}_{3} \mathrm{O}_{5} \mathrm{P}(\mathrm{M}+1)^{+}: 343.1345$, found: 343.1350 .

[3-\{3-(2-Fluoro-4-nitro-pheny])-2-oxo-2,3-dihydro-benzoxazol-6-yl\}aminobutyl]-phosphonic acid diethỵl ester 6a: A flame-dried $100 \mathrm{~mL}$ round-bottom flask under argon was charged with benzophosphonate 5 ( $1.10 \mathrm{~g}$. $3.5 \mathrm{mumol}$ ) and anthydrous $\mathrm{CH}_{3} \mathrm{CN}(20 \mathrm{~mL})$. To this solution was added activated $\mathrm{K}_{3} \mathrm{CO}_{3}(0.72 \mathrm{~g} .5 .25 \mathrm{~nm}$ ol). After being stirred for $5 \mathrm{~min}$ at room temperature, 3.4-difluoronitrobenzene $(0.47$ $\mathrm{mL}, 4.2 \mathrm{mmol}$ ) was added quickly. The reaction mixture was refluxed for $5 \mathrm{hr}$ and quenched with saturated anmonium chloride. This aqueous mixture was extracted with $\mathrm{CH}_{2} \mathrm{Cl}_{2}$ and the combined organic extracts were dried over anhydrous $\mathrm{Na}_{2} \mathrm{SO}_{4}$. After removal of the solvent under reduced pressure. the crude oil was purified by flash column chromatography using a gradient system with methylene chloride and methanol to give the desired product $6 \mathrm{a}$ ( $1.19 \mathrm{~g}$. 2.62 mmol. $75 \%$ ). ${ }^{~} \mathrm{H}$ NMR $\delta 8.21(\mathrm{~m}, 2 \mathrm{H}) .7 .81(\mathrm{~m}, \mathrm{lH})$. $6.70($ dd. $J=2.94,8.58 \mathrm{~Hz}, \mathrm{IH}) .6 .60($ d. $J=2.19 \mathrm{~Hz}, \mathrm{lH})$. 6.43 (dd $J=2.22,8.61 \mathrm{~Hz}, \mathrm{lH}), 4.10(\mathrm{~m}, 4 \mathrm{H}) .3 .53$ (bs. $1 \mathrm{H})$. 1.85 (m. $4 \mathrm{H}$ ). 1.32 (dt. $J=5.31 .6 .96 \mathrm{~Hz} .6 \mathrm{H}), 1.23$ (d. $J=$ $6.24 \mathrm{~Hz}, 3 \mathrm{H}$ ): ${ }^{13} \mathrm{C}$ NMR $\delta 158.02 .153 .39$ (d. $J_{\mathrm{C}-\mathrm{F}}=179.55$ $\mathrm{Hz}) .147 .66\left(\mathrm{~d}, J_{\mathrm{C} \cdot \mathrm{F}}=8.03 \mathrm{~Hz}\right) .145 .26,144.26,128.60(\mathrm{~d}$. $\left.J_{C . \mathrm{F}}=1.8 \mathrm{~Hz}\right) .127 .72\left(\mathrm{~d}, J_{\mathrm{C} \cdot \mathrm{F}}=12.3 \mathrm{~Hz}\right) .120 .56,120.31(\mathrm{~d}$. $\left.J_{\mathrm{C} . \mathrm{F}}=3.68 \mathrm{~Hz}\right), 113.31$ (d. $\left.J_{\mathrm{C} . \mathrm{F}}=24.68 \mathrm{~Hz}\right) .110 .39\left(\mathrm{~d}, J_{\mathrm{C} \cdot \mathrm{F}}=\right.$ $3.68 \mathrm{~Hz}$ ), $109.05 .95 .94,61.67$ (dd. $J_{\mathrm{C} \cdot \mathrm{P}}=1.8,6.15 \mathrm{~Hz}$ ). 49.42 (d, $J_{\text {c. } \mathrm{P}}=15.38 \mathrm{~Hz}$ ), 29.38 (d, $J_{\mathrm{c} . \mathrm{P}}=4.35 \mathrm{~Hz}$ ). 22.32 $\left(\mathrm{d}, J_{\mathrm{C} \cdot \mathrm{P}}=141.38 \mathrm{~Hz}\right) .20 .48,16.49\left(\right.$ dd. $\left.J_{\mathrm{C} . \mathrm{P}}=1.8,6.15 \mathrm{~Hz}\right)$ : ${ }^{31} \mathrm{P}$ NMR $\delta 32.78$; IR $\left(\mathrm{cm}^{-1}\right): 3313.2980 .2210 .1779 .1535$. 1511, 1349; HRFABMS calcd for $\mathrm{C}_{21} \mathrm{H}_{35} \mathrm{~N}_{3} \mathrm{O}_{7} \mathrm{PF}(\mathrm{M}+\mathrm{l})^{-}$: 482.1415 . found: 482.1413 .

[3-\{3-(4-Amino-2-fluoro-phenyl)-2-oxo-2,3-dihydro-benzoxazol-6-yl; aminobutyl]-phosphonic acid diethyl ester 7a: A flame-dried $250 \mathrm{~mL}$ round-bottom flask under argon was charged with nitrobenzophosphonate $6 \mathrm{a}(0.42 \mathrm{~g}, 0.93$ mmol) and anlydrous mixture of THF : $\mathrm{MeOH}(35: 65.100$ $\mathrm{mL})$. To this solution was added ammonium formate $(0.23 \mathrm{~g}$. 3.7 nmol) quickly. After bubbling argon through the reaction solution for $30 \mathrm{~min}$. $\mathrm{Pd} / \mathrm{C}$ (catalyst $1.39 \mathrm{mg}$. 0.014 mmol) was added quickly. The reaction mixture were filtered after 3 hr and concentrated in vacuo to give the crude product. After removal of the solvent under reduced pressure. the crude oil was purified by flash column cluromatography using a gradient solvent system with methylene chloride and methanol to give the desired product 7 a $(0.27 \mathrm{~g} .0 .47 \mathrm{mmol}, 69 \%)$. ${ }^{\mathrm{H}} \mathrm{NMR} \delta 7.22$ (m. $2 \mathrm{H}$ ). 6.56 (m. $4 \mathrm{H}$ ). 6.36 (dd. $J=2.19 .8 .43 \mathrm{~Hz}, \mathrm{lH}), 4.09$ (m. $4 \mathrm{H}), 3.49$ (m. $\mathrm{lH}), 1.82(\mathrm{~m}, 4 \mathrm{H}) .1 .3 \mathrm{l}(\mathrm{dt} . J=5.49 .7 .14 \mathrm{~Hz} .6 \mathrm{H}), 1.20$ (d. $J=6.21 \mathrm{~Hz}, 3 \mathrm{H}) ;{ }^{13} \mathrm{C}$ NMR $\delta 160.21,155.31$ (d, $J_{C . \mathrm{F}}=$ $237.52 \mathrm{~Hz}), 149.24$ (d, $J_{\text {C.F }}=10.4 \mathrm{~Hz}$ ). 144.41. 144.05, $129.34\left(\right.$ d. $\left.J_{C-F}=2.4 \mathrm{~Hz}\right) .123 .06,110.98\left(\mathrm{~d}, J_{C \cdot \mathrm{F}}=2.4 \mathrm{~Hz}\right)$, 110.50 (d. $\left.J_{C \cdot \mathrm{F}}=12.9 \mathrm{~Hz}\right), 109.83\left(\mathrm{~d}, J_{C \cdot \mathrm{F}}=1.2 \mathrm{~Hz}\right) .108 .94$, $102.63\left(\mathrm{~d}, J_{C \cdot \mathrm{F}}=22.2 \mathrm{~Hz}\right) .96 .00,61.64\left(\mathrm{dd} . J_{C \cdot \mathrm{P}}=1.8 .6 .7\right.$ Hz). $49.55\left(\right.$ d. $\left.J_{C \cdot \mathrm{P}}=16.0 \mathrm{~Hz}\right) .29 .37\left(\mathrm{~d} . J_{C . \mathrm{P}}=4.9 \mathrm{~Hz}\right), 22.23$ $\left(\mathrm{d} . J_{c . \mathrm{P}}=140.6 \mathrm{~Hz}\right), 20.49,16.45\left(\mathrm{dd} . J_{C \cdot \mathrm{P}}=1.8,6.1 \mathrm{~Hz}\right):{ }^{31} \mathrm{P}$ NMR $\delta 32.60$ : IR $\left(\mathrm{cm}^{-1}\right)$ : 3320. 2981, 2214, 1790. 1534, 1351; HRFABMS calcd for $\mathrm{C}_{23} \mathrm{FH}_{27} \mathrm{~N}_{2} \mathrm{O}_{3} \mathrm{P}(\mathrm{M}+\mathrm{l})^{+}$. 452.1674 . found 452.1670 .

[3-\{3-(4-Amino-2-fluoro-pheny])-2-oxo-2,3-dihydrobenzoxazol-6-ylaminobutyl]-phosphonic acid 8a: A flame-dried $100 \mathrm{~mL}$ round-bottom flask under argon was charged with aminobenzophosphonate $7 \mathrm{a}(0.2 \mathrm{~g} .0 .5 \mathrm{mmol})$ and anlyydrous $\mathrm{CH}_{2} \mathrm{Cl}_{2}(30 \mathrm{~mL})$. Freshly distilled TMS-Br (1.95 $\mathrm{mL}, 14.9 \mathrm{mmol}$ ) was added through a syringe. The reaction mixture was allowed to stir for $26 \mathrm{lu}$ and quenched with $\mathrm{MeOH}$. This mixture was washed with ethyl acetate several times and then solidified from diethyl ether to get the desired product $8 \mathrm{a}(0.15 \mathrm{~g} .0 .4 \mathrm{mmol} .80 \%)$. ${ }^{1} \mathrm{H}$ NMR (DMSO) $\delta 8.49$ (d. $J=10.1 \mathrm{~Hz} .1 \mathrm{H}$ ). 8.32 (d, $J=8.6 \mathrm{~Hz}$, lH). $8.06(\mathrm{t}, J=8.0 \mathrm{~Hz} . \mathrm{HH}), 7.48$ (bs, IH). 7.15 (bs. $2 \mathrm{H})$, $3.67(\mathrm{~m}, \mathrm{lH}), 1.88(\mathrm{~m} .4 \mathrm{H}) .1 .19(\mathrm{~d}, J=5.9 \mathrm{~Hz} .3 \mathrm{H}) ;{ }^{13} \mathrm{C}$ NMR $\delta 158.17\left(\mathrm{~d}, J_{C \cdot \mathrm{F}}=252.9 \mathrm{~Hz}\right), 154.93,135.16\left(\mathrm{~d} . J_{C . \mathrm{F}}=\right.$ $6.7 \mathrm{~Hz}), 132.64 .130 .83 .129 .40,121.00 .120 .73\left(\mathrm{~d}, J_{\mathrm{C} \cdot \mathrm{F}}=\right.$ $3.7 \mathrm{~Hz}), 1120.12\left(\mathrm{~d}, J_{C \cdot \mathrm{F}}=12.37 \mathrm{~Hz}\right) .113 .20\left(\mathrm{~d}, J_{\mathrm{C} . \mathrm{F}}=22.87\right.$ $\mathrm{Hz}) .111 .81\left(\mathrm{~d}, J_{C \cdot \mathrm{F}}=1.27 \mathrm{~Hz}\right) .107 .43 .60 .68\left(\mathrm{~d}, J_{C \cdot \mathrm{P}}=\right.$ $17.92 \mathrm{~Hz}$ ). 49.67. 23.79 (d. $J_{C \cdot \mathrm{P}}=135.07 \mathrm{~Hz}$ ). 15.86: ${ }^{21} \mathrm{P}$ NMR $\delta 26.41:$ IR $\left(\mathrm{cm}^{-1}\right): 3418.2930,1796,1536.1512$, 1352; HRFABMS calcd for $\mathrm{C}_{17} \mathrm{H}_{19} \mathrm{~N}_{3} \mathrm{O}_{5} \mathrm{PF}(\mathrm{M}+\mathrm{l})^{-}$ 396.1047 , found: 396.1049 .

[3-\{3-(5-Nitro-pyridin-2-y])-2-0xo-2,3-dihydro-benzoxazol-6-yl;aminobutyl]-phosphonic acid diethyl ester 6b: A flame-dried $100 \mathrm{~mL}$ round-bottom flask under $\mathrm{N}_{2}$ was charged with benzophosphonate $5(0.57 \mathrm{~g} .1 .82 \mathrm{mmol})$ and anllydrous $\mathrm{CH}_{3} \mathrm{CN}(20 \mathrm{~mL})$. To this solution was added activated $\mathrm{K}_{2} \mathrm{CO}_{3}(0.38 \mathrm{~g}, 2.72 \mathrm{mmol})$. After being stirred for $5 \mathrm{~min}$ at room temperature, 2 -chloro-5-nitropyridine $(0.35 \mathrm{~g}$. $2.18 \mathrm{mmol}$ ) was added quickly. The reaction mixture was refluxed for $5 \mathrm{hr}$ and theil quenched with saturated ammonium chloride. This aqueous mixture was extracted with $\mathrm{CH}_{2} \mathrm{Cl}_{2}$ and the combined organic extracts were dried over anhydrous $\mathrm{Na}_{2} \mathrm{SO}_{4}$. After removal of the solvent under reduced pressure. the crude oil was purified by flash column chromatography using a gradient solvent system with methylene chloride and methanol to give the desired product 6b $\left(0.48\right.$ g. 1.09 mmol. $60 \%$ ). ${ }^{~} \mathrm{H}$ NMR $\delta 9.31$ (dd. $J=0.9$. $1.0 \mathrm{~Hz}, \mathrm{lH}) .8 .55(\mathrm{~m}, 2 \mathrm{H}), 8.09$ (d. $J=8.8 \mathrm{~Hz} .1 \mathrm{H}), 6.53$ (d. $J=2.4 \mathrm{~Hz}, 1 \mathrm{H}), 6.46(\mathrm{dd}, J=4.4,2.4 \mathrm{~Hz}, 1 \mathrm{H}) .4 .11(\mathrm{~m} .4 \mathrm{H})$, 3.56 (bs. $1 \mathrm{H}$ ). 1.86 (m. $4 \mathrm{H}$ ). 1.33 (dt. $J=5.5,7.2 \mathrm{~Hz}, 6 \mathrm{H}$ ), $1.25(\mathrm{~d} . J=6.2 \mathrm{~Hz} .3 \mathrm{H}):{ }^{13} \mathrm{C}$ NMR $\delta 159.57,153.43,151.85$, 
$145.64,143.88($ d, $J=22.8 \mathrm{~Hz}), 140.68,133.31 .118 .40$. $116.76 .113 .69,108.87,94.54,61.50\left(\mathrm{dd} . J_{C \mathrm{P}}=1.8 .1 .8 \mathrm{~Hz}\right)$. 48.98 (d. $\left.J_{C . \mathrm{P}}=16.1 \mathrm{~Hz}\right) .29 .78\left(\mathrm{~d} . J_{\mathrm{C} . \mathrm{P}}=4.9 \mathrm{~Hz}\right) .22 .06(\mathrm{~d}$. $\left.J_{C . \mathrm{P}}=141.3 \mathrm{~Hz}\right) .20 .21,16.32\left(\mathrm{dd} . J_{\mathrm{C} . \mathrm{P}}=1.2 .1 .8 \mathrm{~Hz}\right) ;{ }^{31} \mathrm{P}$ NMR $\delta$ 32.82: IR $\left(\mathrm{cm}^{-1}\right): 3312,2981,2360.2342,1785$. 1503, 1345; HRFABMS calcd for $\mathrm{C}_{(11} \mathrm{FH}_{2 \leqslant} \mathrm{~N}_{4} \mathrm{O}_{7} \mathrm{P}(\mathrm{M}+1)^{+}$ $: 465.1462$, found 465.1474

[3-\{3-(5-Amino-pyridin-2-y])-2-oxo-2,3-dihydro-benzoxazol-6-yl ;aminobutyl]-phosphonic acid diethyl ester 7b: A flame-dried $250 \mathrm{~mL}$ round-botton flask under $\mathrm{N}_{2}$ was charged with benzophosphonate $6 \mathrm{~b}(0.3 \mathrm{~g}, 0.69 \mathrm{mmol})$ and anhydrous THF : $\mathrm{MeOH}(35: 65,100 \mathrm{~mL})$. To this solution was added anmonium formate $(0.17 \mathrm{~g} .2 .75 \mathrm{mmol})$. After being bubbled for $30 \mathrm{~min}$ with argon $\mathrm{Pd} / \mathrm{C}$ (catalyst $1.39 \mathrm{mg}$. $0.014 \mathrm{mmol}$ ) was added quickly. The reaction mixture was allowed to stir for $3 \mathrm{hr}$, filtered. and concentrated in vacuo to give the crude product. After removal of the solvent under reduced pressure, the crude oil was purified by flash colunn chromatography with a gradient solvent system of methy lene chloride and methanol to give the desired product $7 \mathrm{~b}(0.24 \mathrm{~g}$. $0.59 \mathrm{mmol} .86 \%$ ). ${ }^{1} \mathrm{H}$ NMR $\delta 7.99(\mathrm{~d} . J=2.94 \mathrm{~Hz}, \mathrm{IH}) .7 .84$ $($ d. $J=8.79 \mathrm{~Hz} .1 \mathrm{H}), 7.68(\mathrm{~d}, J=8.61 \mathrm{~Hz} .1 \mathrm{H}), 7.16(\mathrm{dd} . J=$ $2.94,8.76 \mathrm{~Hz} . \mathrm{IH}) .6 .54(\mathrm{~d}, J=2.37 \mathrm{~Hz}, 1 \mathrm{H}) .6 .43(\mathrm{dd} . J=$ $2.19,8.61 \mathrm{~Hz} . \mathrm{lH}), 4.09(\mathrm{~m}, 4 \mathrm{H}), 3.5 \mathrm{l}(\mathrm{m}, \mathrm{lH}), 1.83(\mathrm{~m}$. $4 \mathrm{H}) .1 .32(\mathrm{dt}, J=5.49 .6 .93 \mathrm{~Hz} .6 \mathrm{H}), 1.21(\mathrm{~d} . J=6.24 \mathrm{~Hz}$. $3 \mathrm{H}):{ }^{13} \mathrm{C}$ NMR $\delta 159.73 .153 .67,152.11,145.74,144.12(\mathrm{~d}$. $J=21.6 \mathrm{~Hz}) .140 .94,133.55 .118 .77,116.98 .113 .97$. $109.18 .94 .82,61.44$ (dd. $\left.J_{\mathrm{C} \cdot \mathrm{P}}=1.9 .2 .5 \mathrm{~Hz}\right) .49 .24$ (d. $J_{\mathrm{C} \cdot \mathrm{P}}=$ $15.5 \mathrm{~Hz}), 29.37\left(\mathrm{~d}, J_{\mathrm{C} . \mathrm{P}}=5.0 \mathrm{~Hz}\right), 22.31\left(\mathrm{~d}, J_{\mathrm{C} . \mathrm{P}}=140.6 \mathrm{~Hz}\right)$. $20.46,16.50\left(\mathrm{dd}, J_{\mathrm{C} \mathrm{P}}=1.8 .1 .8 \mathrm{~Hz}\right):{ }^{31} \mathrm{P}$ NMR $\delta 32.76 ; \mathrm{IR}$ $\left(\mathrm{cm}^{-1}\right): 3311.2979,2359.2341 .1785,1503,1340$; HRFABMS calcd for $\mathrm{C}_{3 \mathrm{i}} \mathrm{H}_{37} \mathrm{~N}_{4} \mathrm{O}_{5} \mathrm{P}(\mathrm{M}+1)^{-}: 435.1721$. found: 435.1728 .

[3-\{3-(5-Amino-pyridin-2-y])-2-oxo-2,3-dihydro-benzoxazol-6-yl; aminobutyl]-phosphonic acid 8b: A flamedried $100 \mathrm{~mL}$ round-bottom flask under $\mathrm{N}_{2}$ was charged with benzophosphonate $7 \mathrm{~b}(0.24 \mathrm{~g}, 0.59 \mathrm{mmol})$ and anlydrous $\mathrm{CH}_{2} \mathrm{Cl}_{2}(30 \mathrm{~mL})$. Freshly distilled TMS $-\mathrm{Br}(2.3 \mathrm{~mL}$. $17.7 \mathrm{mmol}$ ) was then added through a syringe. The reaction mixture was allowed to stir for $25 \mathrm{hr}$ and quenched with distilled $\mathrm{MeOH}$ followed by adding of distilled water. This aqueous mixture was washed with ethyl acetate several times and the mixture was solidified from diethyl ether to give the desired product $8 \mathbf{b}(0.2 \mathrm{~g} .0 .57 \mathrm{mmol}, 97 \%) .{ }^{~} \mathrm{H}$ NMR (DMSO) $\delta 9.38$ (d. $J=2.8 \mathrm{~Hz}$. IH). 8.82 (dd. $J=2.9$. $2.7 \mathrm{~Hz} .1 \mathrm{H}), 8.39(\mathrm{~d}, J=9.2 \mathrm{~Hz} .2 \mathrm{H}), 8.23(\mathrm{~d} J=8.8 \mathrm{~Hz}$. lH). 7.08 (m. $2 \mathrm{H}) .3 .66(\mathrm{q}, \mathrm{HH}), 1.71(\mathrm{~m} .4 \mathrm{H}) .1 .17$ (d. $J=$ $6.5 \mathrm{~Hz}, 3 \mathrm{H}) ;{ }^{13} \mathrm{C}$ NMR $\delta$ 159.55. 152.88, $151.23,144.38$. $143.23 .142 .23,139.51,134.37 .118 .94,113.80,102.45$. $94.50,48.68$ (d. $\left.J_{C . \mathrm{P}}=16.7 \mathrm{~Hz}\right) .29 .32,24.14$ (d. $J_{C . \mathrm{P}}=135.8$ Hz). 19.78; ${ }^{31}$ P NMR $\delta 26.59:$ IR $\left(\mathrm{cm}^{-1}\right): 3397.2978 .2462$. 1794, 1580, 1349; HRFABMS calcd for $\mathrm{C}_{16} \mathrm{H}_{19} \mathrm{~N}_{4} \mathrm{O}_{5} \mathrm{P}$ $(\mathrm{M}+\mathrm{l})^{-}: 379.1094$, found: 379.1090 .

[3-\{3-(5-Nitro-4H-[1,2,4]triazo]-3-yl)-2-oxo-2,3-dihydrobenzoxazol-6-yl;aminobutyl]-phosphonic acid diethyl ester 6c: A flame-dried $100 \mathrm{~mL}$ round-bottom flask under $\mathrm{N}_{2}$ was charged with benzophosphonate $5(0.57 \mathrm{~g} .1 .18$ $\mathrm{mmol})$ and anhydrous $\mathrm{CH}_{5} \mathrm{CN}(20 \mathrm{~mL})$. To this solution was added activated $\mathrm{K}_{2} \mathrm{CO}_{3}(0.38 \mathrm{~g} .2 .72 \mathrm{mmol})$ After being stirred for $5 \mathrm{~min}$ at room temperature. 5-bromo-3-nitro1.2.4-triazole $(0.42 \mathrm{~mL} .2 .18 \mathrm{mmol})$ was added quickly. The reaction mixture was refluxed for 5 hr and quenched with distilled water and ammonium chloride. This aqueous mixture was extracted with $\mathrm{CH}_{2} \mathrm{Cl}_{2}$ and then the combined organic extracts were dried over anhydrous $\mathrm{Na}_{2} \mathrm{SO}_{4}$. After removal of the solvent under reduced pressure. the crude oil was purified by flash column chromatograply using a gradient solvent system with metlylene chloride and methanol to give the desired product $6 \mathrm{c}(0.53 \mathrm{~g} .1 .13 \mathrm{mmol}$, $63 \%$ ). ${ }^{1} \mathrm{H}$ NMR $\delta 9.18$ (bs. lH). 6.79 (d. $J=8.4 \mathrm{~Hz}, \mathrm{lH}$ ), $6.50(\mathrm{~d}, J=2.0 \mathrm{~Hz} . \mathrm{IH}), 6.36(\mathrm{dd}, J=2.2 .2 .2 \mathrm{~Hz} . \mathrm{IH}) .4 .11$ (m. $5 \mathrm{H}) .3 .50(\mathrm{~m}, \mathrm{lH}) .1 .84(\mathrm{~m}, 4 \mathrm{H}), 1.33$ (dt. $J=3.3 .7 .0$ Hz. $6 \mathrm{H}) .1 .20(\mathrm{~d}, J=6.2 \mathrm{~Hz} .3 \mathrm{H}) ;{ }^{13} \mathrm{C}$ NMR $\delta 155.72$, $145.29,143.73$. 139.28, 120.63, 114.07. 110.31. 109.17, $96.20,61.82\left(\right.$ d. $\left.J_{C . \mathrm{P}}=6.2 \mathrm{~Hz}\right) .49 .56\left(\right.$ d. $\left.J_{C . \mathrm{P}}=16.1 \mathrm{~Hz}\right)$, $29.20\left(\right.$ d. $J_{C . \mathrm{P}}=5.0 \mathrm{~Hz}$ ). $22.09\left(\mathrm{~d}, J_{C \cdot \mathrm{P}}=140.7 \mathrm{~Hz}\right.$ ). 20.43 . $16.45\left(\mathrm{dd} . J_{C . \mathrm{P}}=1.2 .1 .2 \mathrm{~Hz}\right):{ }^{31} \mathrm{P}$ NMR $\delta 33.26:$ IR $\left(\mathrm{cm}^{-1}\right)$ : 3355. 2979. 2370, 2340. 1763, 1498. 1026; HRFABMS calcd for $\mathrm{C}_{17} \mathrm{H}_{23} \mathrm{~N}_{6} \mathrm{O}_{7} \mathrm{P}(\mathrm{M}+\mathrm{l})^{+}: 455.1372$, found 455.1376 .

[3-\{3-(5-Amino-4H-[1,2,4]triazol-3-yl)-2-oxo-2,3-dihydrobenzoxazol-6-yl;amino-butyl]-phosphonic acid diethyl ester 7c: A flame-dried $250 \mathrm{~mL}$ round-bottom flask under $\mathrm{N}_{2}$ was charged with benzophosphonate $6 \mathrm{c}(0.47 \mathrm{~g} .0 .1$ mmol) and anlydrous THF : MeOH $(35: 65.100 \mathrm{~mL})$. To this solution was added ammonium formate $(0.25 \mathrm{~g}, 0.4$ mmol). After being bubbled for $30 \mathrm{~min}$ for argon. $\mathrm{Pd} / \mathrm{C}$ (catalyst $1.39 \mathrm{mg} .0 .014 \mathrm{mmol}$ ) was added quickly. The reaction mixture was allowed to stir for $3 \mathrm{hr}$. filtered, and concentrated in vacto to give the crude product. After removal of the solvent under reduced pressure. the crude oil was purified by flash column chromatograply using a gradient solvent system with methylene chloride and methanol to give the desired product $7 \mathrm{c}(0.46 \mathrm{~g}$. $1.04 \mathrm{mmol}$, $69 \%) .{ }^{1} \mathrm{H}$ NMR $\delta 8.78(\mathrm{~s}, \mathrm{lH}), 6.78(\mathrm{~d}, J=8.43 \mathrm{~Hz}, \mathrm{lH})$, $6.50($ d. $J=2.19 \mathrm{~Hz} .1 \mathrm{H}), 6.35(\mathrm{dd}, J=2.19 .8 .43 \mathrm{~Hz}, \mathrm{lH})$. $4.11(\mathrm{~m}, 4 \mathrm{H}) .3 .48(\mathrm{~m}, \mathrm{lH}) .1 .85(\mathrm{~m}, 4 \mathrm{H}) .1 .32(\mathrm{dt} . J=3.84$. $6.96 \mathrm{~Hz}, 6 \mathrm{H}) .1 .20(\mathrm{~d}, J=6.24 \mathrm{~Hz}, 3 \mathrm{H}):{ }^{13} \mathrm{C}$ NMR $\delta 155.94$, $145.23,143.49 .139 .25,120.89,114.03 .110 .37,109.26$, $96.18,61.83$ (d. $J_{C . \mathrm{P}}=6.8 \mathrm{~Hz}$ ). 49.59 (d. $J_{C . \mathrm{P}}=16.1 \mathrm{~Hz}$ ), $29.62\left(\right.$ d. $J_{\text {C.P }}=4.3 \mathrm{~Hz}$ ). $22.00\left(\mathrm{~d} . J_{C \cdot \mathrm{P}}=140.7 \mathrm{~Hz}\right.$ ). 20.33 . $16.40\left(\right.$ dd. $\left.J_{C . \mathrm{P}}=1.2 .1 .2 \mathrm{~Hz}\right)$ : ${ }^{31} \mathrm{P}$ NMR $\delta 30.2 \mathrm{l}:$ IR $\left(\mathrm{cm}^{-1}\right)$ : 3366. 2980.2360. 1764. 1496, 1027.

[3-\{3-(5-Amino- $4 H-[1,2,4]$ triazol-3-yl)-2-oxo-2,3-dihydrobenzoxazol-6-yl;amino-butyl]-phosphonic acid 8c: A flame-dried $100 \mathrm{~mL}$ round-bottom flask under $\mathrm{N}_{2}$ was charged with benzophosphonate $7 \mathrm{c}(0.4 \mathrm{~g} .0 .91 \mathrm{mmol})$ and anhydrous $\mathrm{CH}_{2} \mathrm{Cl}_{2}(30 \mathrm{~mL})$. Freshly distilled TMS-Br (3.6 $\mathrm{mL}, 27.2 \mathrm{mmol}$ ) was then added through a syringe. The reaction mixture was allowed to stir for $26 \mathrm{hr}$ and quenched with $\mathrm{MeOH}$ followed by adding of distilled water. This aqueous mixture was washed with ethyl acetate several times, and the misture was solidified from dietlyl ether to give the desired product $8 \mathrm{c}(0.21 \mathrm{~g}, 0.64 \mathrm{mmol}, 70 \%) .{ }^{1} \mathrm{H}$ NMR (DMSO) $\delta 11.06(\mathrm{bs}, 1 \mathrm{H}) .6 .76(\mathrm{~d} . J=8.4 \mathrm{~Hz} . \mathrm{lH}$ ), 6.52 (s. $\mathrm{lH}$ ), 6.35 (dd. $J=2.2 .2 .2 \mathrm{~Hz}, \mathrm{lH}) .3 .82(\mathrm{~m} . \mathrm{lH}$ ), 
$3.37(\mathrm{~m} . \mathrm{HH}) .1 .59(\mathrm{~m}, 4 \mathrm{H}), 1.05(\mathrm{~d} . J=6.0 \mathrm{~Hz}, 3 \mathrm{H}):{ }^{13} \mathrm{C}$ NMR $\delta 154.70,144.64$. 144.54. 140.22, 119.54. 110.01. $108.00,94.64 .64 .90 .48 .4 \mathrm{I}\left(\mathrm{d} . J_{C . \mathrm{P}}=18.6 \mathrm{~Hz}\right) .19 .56 .16 .50$. 16.46, 15.15: ${ }^{31} \mathrm{P}$ NMR $\delta$ 26.84: IR $\left(\mathrm{cm}^{-1}\right): 3417,2978$. $1769,1638,1505,1040$ : HRFABMS calcd for $\mathrm{C}_{13} \mathrm{H}_{17} \mathrm{~N}_{6} \mathrm{O}_{5} \mathrm{P}$ $(\mathrm{M}+\mathrm{I})^{-}: 369.1000$. found: 369.1008 .

Acknowledgement. This work has been supported by a grant from the Korea Science and Engineering Foundation (Grant R01-2001-000-00214-0).

\section{References}

1. Slee, A. M.: Wuonola, M. A.: McRipley. R. I.: Zajac I: Zawada. M. J.: Batholomew. P. T.: Gregory. W. A.: Forbes. M. Abstracts of 27h hiterscience Conference on Antmicrobial Agents and Chemotherapv: New York. Oet. 4-7. 1987: abstract No. 244. ii. Bricker. S. I.: Hutchison. D. K.: Barbachyin. M. R.: Manninen. P. R.: Vlanowicz, D. A.: Gammon, S. A.: Grega. K. C: Hendges, S. K.: Toops, D. S.: Ford. C. V: Zurenko. G. E. J. hed Chent 1996. 39.673 .

2. Brumfitt. W: Hamilton-Miller. J. M. T. Drugs Exp. Clm. Res. 1994..11:215

3. (a) Brickiner S. J. Cur Pham Des, 1996, 2. 175. (b) Brumfitt. W. Hamilton-Miller. J. M. T. Drugs Exp. Chin. Res. 1994 .11:
215

4. Jones. R. N.: Sader. H. S. Challenges Infect Dis. 1993. 1. I

5. (a) Fugitt. R. B; Luckenbaugh, R. W. U.S. Patent $1,128,65$ t. 1996. (b) Eustice, D. C.; Feldman. P. A.; Zajac. I.; Slee. A. M. Antinticrob. Agents Chemother. 1988. $32,1218$.

6. (a). Ucar. H.: Van depooter1. K.: Cacciaguerra. S.: Spampinato. S.: Stables. T. P.: Depovere. P.: Masereel. B.: Isa. M.: Delarge. T.: Poupaert. J. H. J. Hed Chem 1998. 41. 1138. (b). Pashov. D.: Bakurdzhiev. A.; Kalcheva, V; Simov. D.: Kamenova. L: Boicheva. K. H. Fom matsiva 1975, 25,61.

7. (a) Beers, S. A.: Schwender, C. F.: Loughney, D. A.; Malloy, E.: Demarest. K.: Jordat1. J. Bioong Med. Chem. 1996. f. 1693. (b) Kase. H.: Yamamoto. M.: Koguchi. T.: Okachi. R.: Kasai. M. Shirahata. K.: Kawamoto. I.: Shuto. K.: Karasawa. A.: Deguchi. T.: Nakayama. K. Eur. Pot. Appl. EP 0061172 Al. 1982: Chem. Abstr. 1983, 98. 107793. (c) Jung. K. Y; Hwang, J. M. Bull. Korean Chem. Soc. 2002. 23(12), 1848.

8. (a) Jung. K. Y.: Lee. M. Y.: McClure. C. K. Phosphorts, Sulfur: and Silicon 1999. 147. 141. (b) McClure. C. K.: Hausel. R. C.: Hansen. K. B.: Grote. C. W.: Jung. K. Y. Phosphonts. Sulfur and Silicon 1996. Hll, 63

9. (a) McClure. C. K.: Jung, K. Y. J. Org. Chem. 1991, 56, 867. (b) MoClure. C. K.: Grote. C. W. Terahedron Letf. 1991, 32, 5313. (c) Jung. K. Y.: Lee. M. Y. J. Ind \& Eng. Chem. 1999. 5(3). 224.

10. Methods for dilution antimicrobial susceptibility tests for bacteria that grow aerobically: approved standard. 6th ed: NCCLS document M7-A6 (ISBN 1-56238-486-4). NCCLS: Pennsvivania. USA, 2003. 